\title{
Nanopores of carbon nanotubes as practical hydrogen storage media
}

\author{
Sang Soo Han, Hyun Seok Kim, Kyu Sung Han, Jai Young Lee, \\ Hyuck Mo Lee, and Jeung Ku Kang ${ }^{\text {a) }}$ \\ Department of Materials Science and Engineering, KAIST, Daejeon 305-701, Republic of Korea \\ Seong Ihl Woo \\ Department of Chemical and Biomolecular Engineering, KAIST, Daejeon 305-701, Republic of Korea
}

Adri C. T. van Duin and William A. Goddard III

Materials and Process Simulation Center, California Institute of Technology, California 91125

(Received 2 June 2005; accepted 12 October 2005; published online 16 November 2005)

\begin{abstract}
We report on hydrogen desorption mechanisms in the nanopores of multiwalled carbon nanotubes (MWCNTs). The as-grown MWCNTs show continuous walls that do not provide sites for hydrogen storage under ambient conditions. However, after treating the nanotubes with oxygen plasma to create nanopores in the MWCNTs, we observed the appearance of a new hydrogen desorption peak in the 300-350 K range. Furthermore, the calculations of density functional theory and molecular dynamics simulations confirmed that this peak could be attributed to the hydrogen that is physically adsorbed inside nanopores whose diameter is approximately $1 \mathrm{~nm}$. Thus, we demonstrated that $1 \mathrm{~nm}$ nanopores in MWCNTs offer a promising route to hydrogen storage media for onboard practical applications. (C) 2005 American Institute of Physics. [DOI: 10.1063/1.2133928]
\end{abstract}

Since the first experimental report of Dillon et al. ${ }^{1}$ on hydrogen storage in carbon nanotubes (CNTs), there have been many experimental and theoretical studies on the hydrogen uptake capacity in various CNT structures. ${ }^{2-4}$ Nanotubes with high surface-to-volume ratios are ideal for the fast kinetics of hydrogenation and dehydrogenation. However, despite this advantage, the use of pristine CNTs as hydrogen storage media is costly; furthermore, due to the low adsorption temperature (80-150 $\mathrm{K}$ at $1 \mathrm{bar}$ ) of hydrogen on pristine CNTs, an intensive cryogenic process is needed for cooling. Consequently, there remains a great challenge in storing hydrogen under ambient conditions that are ideal for practical applications. Here, we report that $1 \mathrm{~nm}$ nanopores in CNTs offer a promising solution to this challenge.

To synthesize multiwalled CNTs (MWCNTs), we used thermal chemical vapor deposition (CVD) and microwave plasma-enhanced CVD (PECVD). In the thermal CVD, we synthesized the nanotubes by using a floating catalyst method under a $0.04 \mathrm{~g} / \mathrm{ml}$ solution of ferrocene in xylene. After obtaining the MWCNTs on a $\mathrm{SiO}_{2}$ substrate, we etched them immediately by using atmospheric plasma, and for the etching gas, we added $2 \%$ of $\mathrm{O}_{2}$ to $\mathrm{He}$. On the other hand, for the PECVD, we first deposited a $50 \mathrm{~nm}$ thick cobalt layer on a $\mathrm{Si}$ substrate by using rf-magnetron sputtering. For the gas source, we used a mixture of $\mathrm{H}_{2}(89.9 \%), \mathrm{CH}_{4}(0.1 \%)$, and $\mathrm{O}_{2}(10 \%)$ with a pressure of 30 Torr and a temperature of $750{ }^{\circ} \mathrm{C}$. As a result, we formed open-tip MWCNTs.

We determined the hydrogen desorption properties by analyzing thermal desorption spectra (TDS). For this analysis, we degassed the sample at $300{ }^{\circ} \mathrm{C}$ for $48 \mathrm{~h}$ under a vacuum below $10^{-3}$ Torr, and then charged pure hydrogen (99.999\%) under $40 \mathrm{~atm}$ at $300 \mathrm{~K}$. Next, we placed the sample in a liquid nitrogen-cooled cryostat with a programmable heating supply apparatus. To detect the hydrogen that evolved from the MWCNTs, we used a gas chromatograph

\footnotetext{
a) Author to whom correspondence should be addressed; electronic mail: jeungku@kaist.ac.kr
}

with a temperature scanning range of $80-673 \mathrm{~K}$ and a rate of $3 \mathrm{~K} / \mathrm{min}$.

In addition, the density functional theory (DFT) ${ }^{5,6}$ and the molecular dynamics simulation methods using a reactive force field (ReaxFF $)^{7-9}$ are used to determine hydrogen storage and desorption properties of MWCNTs, where the MD simulation was first performed under an NVT ensemble for 400000 steps and then under an NVE ensemble for 400000 steps.

Figures 1(a) (before plasma etching) and 1(b) (after plasma etching) show hydrogen desorption spectra of the open-tip MWCNTs, prepared by thermal CVD; the outer diameters of the nanotubes are in the range of $20-50 \mathrm{~nm}$ and the inner diameters are in the range of $10-20 \mathrm{~nm}$. We found that the plasma etching opens the MWCNTs tip. Before the atmospheric plasma etching, we observed that the hydrogen molecules evolved at only $100-150 \mathrm{~K}$ and that $4.9 \mathrm{wt} \%$ of hydrogen was released from the peak. In contrast, as shown in Fig. 1(b), we observed an ambient temperature peak at $300-350 \mathrm{~K}$ after the plasma etching. In this case, $0.6 \mathrm{wt} \%$ of hydrogen was released near an ambient temperature. Figure 1(c) shows hydrogen desorption spectra from MWCNTs synthesized by PECVD with oxygen plasma. Two different peaks occurred at temperature ranges of $100-200 \mathrm{~K}$ and $300-350 \mathrm{~K}$. We obtained a total integrated amount of 1.8 wt $\%$ hydrogen from the first peak and $2.0 \mathrm{wt} \%$ hydrogen from the second peak. In Figs. 1(a) and 1(c), the peak in the low-temperature range evolved at $100-150 \mathrm{~K}$ and the second peak evolved in the range of 300-350 K, which consequently implies that the hydrogen adsorption site for each peak is the same irrespective of the synthesis method. And Fig. 1(d) shows a high-resolution transmission electron microscope (HRTEM) image of an open-tip MWCNT synthesized by PECVD. The outer diameters ranged from 10 to $30 \mathrm{~nm}$ and the inner diameters ranged from 5 to $15 \mathrm{~nm}$. In our MWCNT sample, the defective slits, shown as circles in Fig. 1(d), were estimated to be approximately $1 \mathrm{~nm}$ in the tube wall. Our 


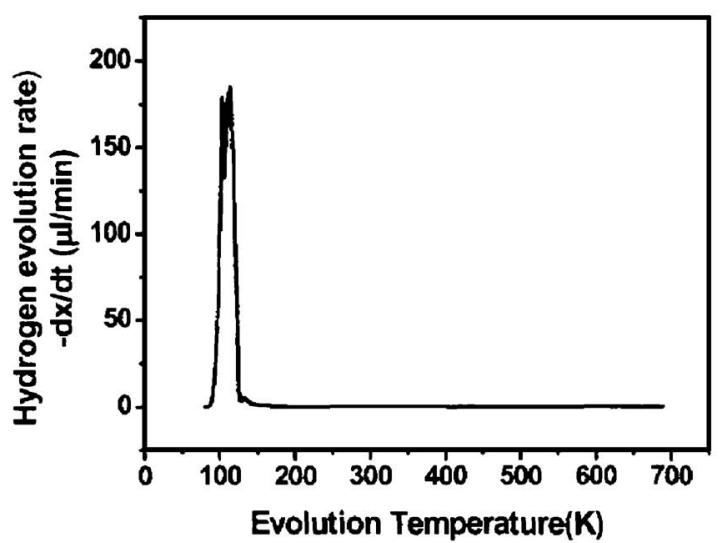

(a)

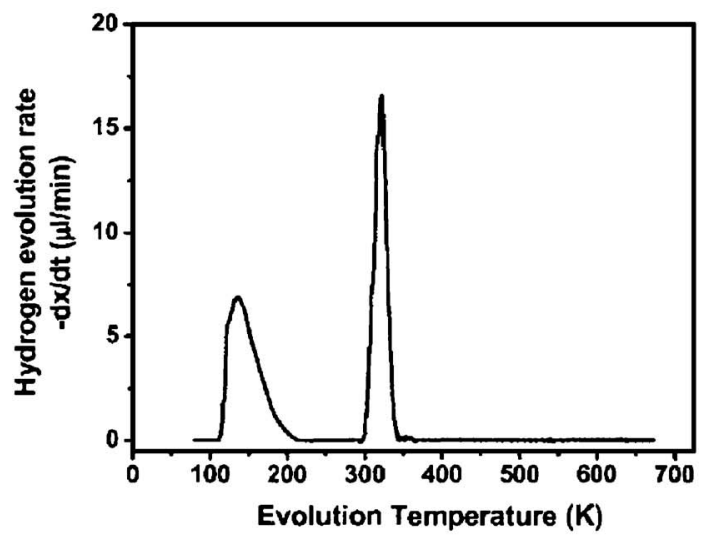

(c)

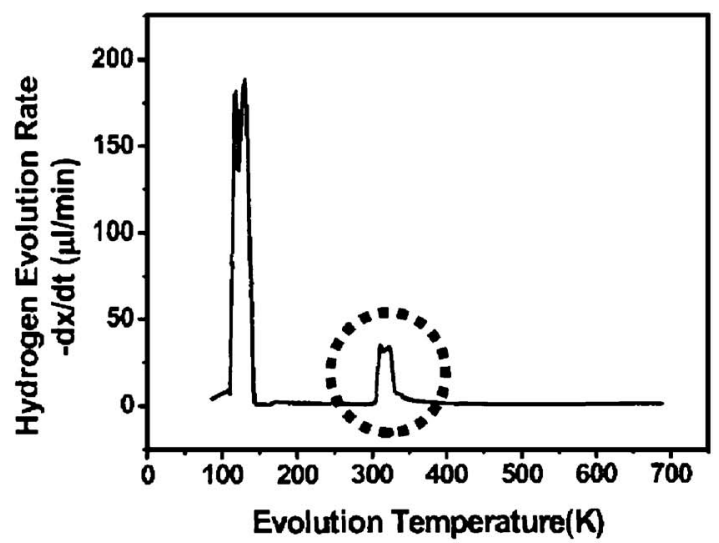

(b)

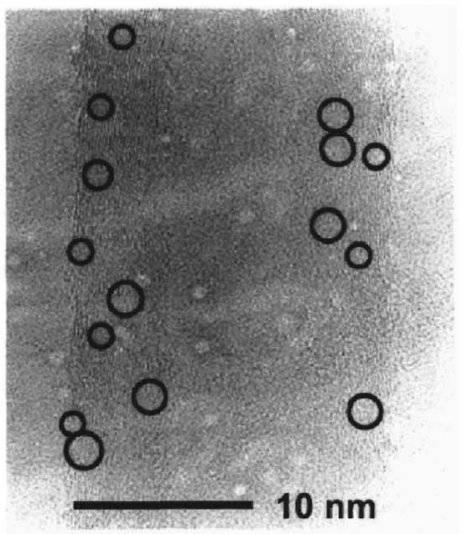

(d)

FIG. 1. TDS of MWCNTs synthesized by thermal CVD (a) before and (b) after atmospheric plasma treatment, (c) TDS of MWCNTs synthesized by PECVD and (d) a HRTEM image for MWCNTs synthesized by PECVD where the circles indicate the nanopores with $\sim 1 \mathrm{~nm}$ size existing in the MWCNT wall. of Gao et al. ${ }^{10}$ and Hou et al. ${ }^{11}$

The TDS data of Figs. 1(a)-1(c) could not give atomistic details for the hydrogen adsorption sites. Hence, we used DFT and MD simulations to gain a better understanding of the hydrogen desorption mechanisms of the nanopores in the MWCNTs. The hydrogen that is chemisorbed with CNT cannot be released near room temperature. ${ }^{3}$ Thus, the peaks in Figs. 1(a)-1(c) involve hydrogen that has been physically adsorbed in the MWCNT. Table I summarizes the physisorption energies of hydrogen on various sites of $(10,0)$ CNT. The exothermic enthalpy of about $1 \mathrm{kcal} / \mathrm{mol}$ indicates that, from van't Hoff's equation, ${ }^{12}$ the hydrogen adsorption on or in pristine CNTs is possible only at temperatures ranging from $50-80 \mathrm{~K}$ at $0.1 \mathrm{MPa}$. In addition, the vacancy and edge carbon atoms can enhance the exothermic enthalpies of hydrogen adsorption; thus, the adsorption temperatures in these

TABLE I. DFT results for the change of heat $(\mathrm{kcal} / \mathrm{mol})$ in hydrogen adsorption on several sites of the CNT and estimated adsorption temperatures (K) at 0.1 and $4 \mathrm{MPa}$. Cases (A), (B), (C), (D), and (E) mean physisorption of $\mathrm{H}_{2}$ on the exterior nanotube wall, on the interior nanotube wall, on a vacancy in the exterior wall, on a vacancy in the interior wall, and on edge

\begin{tabular}{ccrrrr}
\hline \hline Case & (A) & \multicolumn{1}{c}{$(\mathrm{B})$} & \multicolumn{1}{c}{$(\mathrm{C})$} & \multicolumn{1}{c}{$(\mathrm{D})$} & \multicolumn{1}{c}{$(\mathrm{E})$} \\
\hline Adsorption energy & -0.8 & -1.4 & -2.1 & -2.6 & -2.4 \\
Temperature (0.1 MPa) & 47.0 & 82.0 & 124.0 & 153.0 & 141.0 \\
Temperature (4.0 MPa) & 83.0 & 145.0 & 218.0 & 270.0 & 249.0 \\
\hline
\end{tabular}

observation of these nanopores is consistent with the findings atoms on the CNT tip, respectively.

sites increase to $120-150 \mathrm{~K}$ at $0.1 \mathrm{MPa}$. And, at $4.0 \mathrm{MPa}$, hydrogen adsorption may occur on vacancy sites at temperatures up to $200 \mathrm{~K}$. Through MD simulations at different temperatures, we determined the hydrogen adsorption and desorption properties from the open tips of the CNTs. $\mathrm{H}_{2}$ is incapable of being stored inside the $(10,0)$ CNT tip up to 100 $\mathrm{K}$, and this finding is consistent with the DFT results in Table I. The $\mathrm{H}_{2}$ molecule, which is sucked into the open tip within 2.0 ps because of the nanocapillary phenomenon, moves from the CNT tip to other sites via several movements, after which it is finally stored inside the tube. The $\mathrm{H}_{2}$ molecule stored in the open-tip does not escape from the nanotube,

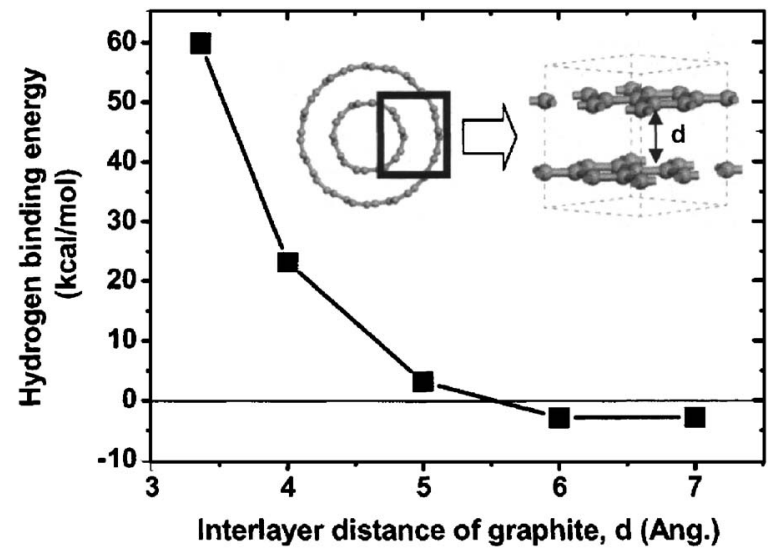

FIG. 2. DFT results on hydrogen adsorption energies in the interlayer space of the graphite as a function of the interlayer distance. Downloaded 14 Dec 2005 to 131.215.225.171. Redistribution subject to AIP license or copyright, see http://apl.aip.org/apl/copyright.jsp 


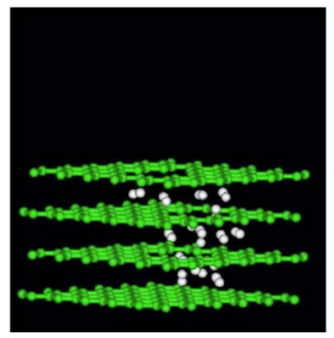

(a)

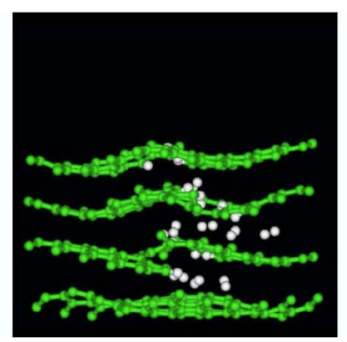

(b)

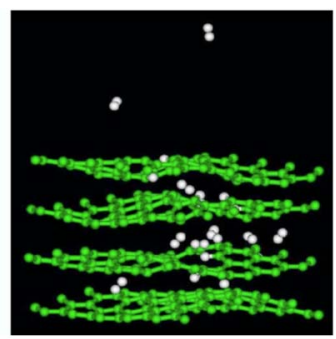

(c) even after $200 \mathrm{ps}$ in the NVT ensemble condition; nor does it escape after an additional 200 ps in the NVE ensemble. Regarding the desorption behavior of the hydrogen molecules stored inside the CNT, we found that the hydrogen escapes from the CNT at $150 \mathrm{~K}$. To determine how the CNT diameter affects the adsorption and desorption of hydrogen, we also considered a $(10,10)$ CNT with a diameter of $13.6 \AA$, which is almost twice as large as the diameter of the $(10,0)$ CNT. The $\mathrm{H}_{2}$ molecule is absorbed inside the tube at $100 \mathrm{~K}$ and desorbs at $140 \mathrm{~K}$, thereby indicating that the hydrogen adsorption and desorption temperatures are similar for various CNT diameters whenever the diameters are much larger than $1 \mathrm{~nm}$. In exploring the mechanisms of hydrogen adsorption and desorption on the exterior wall, we found that $\mathrm{H}_{2}$ was adsorbed on the exterior wall of the $(10,0)$ SWCNT at $60 \mathrm{~K}$ and released from the CNT at $80 \mathrm{~K}$ irrespective of the CNT diameter. We conclude therefore that the first peak that was experimentally observed at $100-200 \mathrm{~K}$ was mostly due to the desorption of the hydrogen stored in the MWCNT hole. Our analysis of Raman spectra also indicates that the crystallinity (degree of graphitization) of the MWCNTs prepared by PECVD is lower than that of the MWCNTs prepared by thermal CVD. Thus, we consider that the pure graphene wall area of the nanotubes synthesized by PECVD is smaller than that synthesized by thermal CVD. This graphene area is proportional to the amount of hydrogen adsorbed at the lower peak. Consequently, as reflected by the first peak, the hydrogen storage capacity of the MWCNTs prepared by thermal CVD is higher than that of MWCNTs prepared by PECVD. In addition, the low-temperature peak of Fig. 1(c) is broader than the corresponding peaks of Figs. 1(a) and 1(b), possibly because the velocity distribution of the hydrogen that evolved from MWCNTs prepared by PECVD is more random than the velocity distribution of the hydrogen that evolved from MWCNTs prepared by thermal CVD.

We also investigated why the second peaks in Figs. 1(b) and 1(c) occurred at around room temperature. Other researchers have suggested that hydrogen molecules could be stored in the intertube hollow of MWCNTs. ${ }^{2,13}$. In the DFT calculations (see Fig. 2), we used the graphite layers to model the intertube layers in the MWCNT. At an interlayer distance of $3.36 \AA$, we determined that hydrogen is thermodynamically unstable because the binding energy of the hydrogen in the interlayer is endothermic by $59.94 \mathrm{kcal} / \mathrm{mol}$. This result implies that the $\mathrm{H}_{2}$ molecule cannot plausibly be stored in the intertube of a normal MWCNT. Thus, we cannot attribute the second peak near room temperature, as shown in Figs. 1(b) and 1(c), to the hydrogen stored in the intertube of the MWCNT. Figure 2, on the other hand, shows that hydrogen could be stable in the interlayer space of the graphite or in the intertube of the MWCNT before the interlayer or intertube distances become $6 \AA$ because the binding energy of the hydrogen adsorption is exothermic by $2.84 \mathrm{kcal} / \mathrm{mol}$.

As seen in Fig. 3(a), we also used four layers of a graphite structure (trigonal with $a=b=14.66 \AA, c=40 \AA$, and $\gamma$ $=60^{\circ}$ ) for the initial structure of the MD simulation, where a nanopore of about $1 \mathrm{~nm}$ into the graphite was introduced. At the start, we set $15 \mathrm{H}_{2}$ molecules to be stored inside the nanopore. To study the desorption temperature of the hydrogen molecules initially stored in the nanopore, we used an NVT-MD simulation for the initial system of Fig. 3(a) at four simulation temperatures of $200 \mathrm{~K}, 250 \mathrm{~K}, 300 \mathrm{~K}$, and $350 \mathrm{~K}$. Below $250 \mathrm{~K}$, as depicted in Figs. 3(b) and 3(c), we found that none of the hydrogen stored in the nanopore was released until 200 ps of the NVE-MD simulation; moreover, the hydrogen only started to escape from the nanopore at 300 K. Figure 3 therefore indicates that the second peaks at room temperature in Figs. 1(b) and 1(c) are attributed to the hydrogen desorption from the nanopores of about $1 \mathrm{~nm}$.

In conclusion, we find that any nanopores of about $1 \mathrm{~nm}$ that exist in MWCNT walls are available for hydrogen adsorption sustainable up to room temperature. Thus, we have demonstrated that nanopores of this size offer a promising route to hydrogen storage sites for practical hydrogen storage media.

This research was supported by the research program of KOSEF (Grant No. R012005000103330) and the Center for Ultramicrochemical Process Systems (CUPS) sponsored by KOSEF (2005).

${ }^{1}$ A. C. Dillon, K. M. Jones, T. A. Bekkedahl, C. H. Klang, D. S. Bethune, and M. J. Heben, Nature (London) 386, 377 (1997).

${ }^{2}$ A. Chambers, C. Park, R. Terry, and K. Baker, J. Phys. Chem. B 102, 4254 (1998).

${ }^{3}$ H. Lee, Y.-S. Kang, S.-H. Kim, and J.-Y. Lee, Appl. Phys. Lett. 80, 577 (2002).

${ }_{5}^{4}$ S. S. Han and H. M. Lee, Carbon 42, 2169 (2004).

${ }^{5}$ J. P. Perdew and Y. Wang, Phys. Rev. B 45, 13244 (1992).

${ }^{6}$ B. Delly, J. Chem. Phys. 113, 7756 (2000).

${ }^{7}$ A. C. T. van Duin, S. Dasgupta, F. Lorant, and W. A. Goddard III, J. Phys. Chem. A 105, 9396 (2001).

${ }^{8}$ K. D. Nielson, A. C. T. van Duin, J. Oxgaard, W.-Q. Deng, and W. A. Goddard III, J. Phys. Chem. A 107, 3803 (2003).

${ }^{9}$ S. S. Han, J. K. Kang, H. M. Lee, A. C. T. van Duin, and W. A. Goddard III, Appl. Phys. Lett. 86, 203108 (2005).

${ }^{10}$ H. Gao, X. B. Wu, J. T. Li, G. T. Wu, J. Y. Lin, K. Wu, and D. S. Xu, Appl. Phys. Lett. 83, 3389 (2003).

${ }^{11}$ P.-X. Hou, S.-T. Xu, Z. Ying, Q.-H. Yang, C. Liu, and H.-M. Cheng, Carbon 41, 2471 (2003).

${ }^{12}$ The van't Hoff equation is given as $\ln p=-\Delta H / R T+\Delta S / R$, where $\Delta H$ is the heat of adsorption, $R$ is the gas constant, and $\Delta S$ is the change in entropy. We found that the $\Delta S$ is about $-0.02 \mathrm{kcal} / \mathrm{molK}$ at ambient conditions.

${ }^{13}$ C. Park, P. E. Anderson, A. Chambers, C. D. Tan, R. Hidalgo, and N. M. Rodriguez, J. Phys. Chem. B 103, 10572 (1999). 